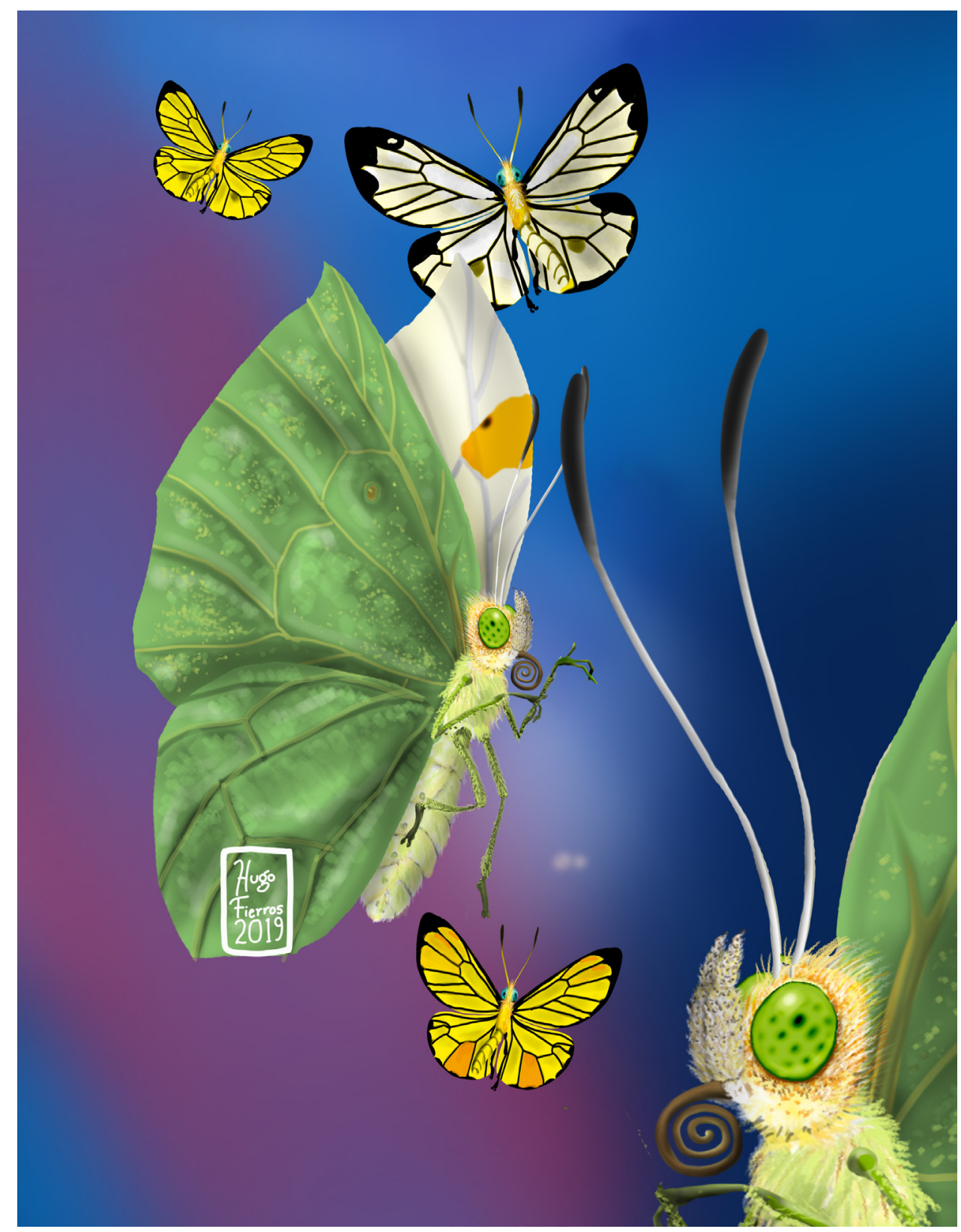

Dugesiana, Año 26, No. 2, julio 2019-diciembre 2019 (segundo semestre de 2019), es una publicación semestral, editada por la Universidad de Guadalajara, a través del Centro de Estudios en Zoología, por el Centro Universitario de Ciencias Biológicas y Agropecuarias. Camino Ramón Padilla Sánchez \# 2100, Nextipac, Zapopan, Jalisco, Tel. 37771150 ext. 33218, http://148.202.248.171/dugesiana/index.php/DUG/index, glenusmx@gmail.com. Editor responsable: José Luis Navarrete Heredia. Reserva de Derechos al Uso Exclusivo 04-2009-062310115100203, ISSN: 2007-9133, otorgados por el Instituto Nacional del Derecho de Autor. Responsable de la última actualización de este número: José Luis Navarrete Heredia, Editor y Ana Laura González-Hernández, Asistente Editorial. Fecha de la última modificación 25 de julio 2019, con un tiraje de un ejemplar.

Las opiniones expresadas por los autores no necesariamente reflejan la postura del editor de la publicación.

Queda estrictamente prohibida la reproducción total o parcial de los contenidos e imágenes de la publicación sin previa autorización de la Universidad de Guadalajara. 


\section{Descripción de Lycomesus llorentei gen. et sp. nov., (Coleoptera: Lycini) de San Luis Potosí, México}

\section{Description of Lycomesus llorentei gen. et sp. nov., (Coleoptera: Lycidae: Lycini) from San Luis Potosí, Mexico}

\section{Santiago Zaragoza-Caballero ${ }^{1}$ y Mireya González-Ramírez ${ }^{1}$}

Departamento de Zoología, Instituto de Biología, Universidad Nacional Autónoma de México, Apartado Postal 70-153, 04510 CdMx, México. ${ }^{1 E m a i l: ~ z a r a g o z a @ i b . u n a m . m x ~}$

\section{RESUMEN}

Se describe Lycomesus llorentei gen. nov., sp. nov. (Coleoptera: Lycidae: Lycini), procedente de San Luis Potosí, México. Se presenta un cuadro comparativo con los caracteres diagnósticos entre los miembros de Lycini en Norteamérica: Lycus, Lycostomus, Lyconotus y Lycomesus.

Palabras clave: Insecta, diversidad, especie nueva, taxonomía, Lycidae, México.

\section{ABSTRACT}

Lycomesus llorentei gen. et sp. nov. (Coleoptera: Lycidae: Lycini) is described from San Luis Potosí, Mexico. A comparative table with the diagnostic characters for the members of Lycini from North America, Lycus, Lycostomus, Lyconotus and Lycomesus, is presented.

Key words: Insecta, diversity, new species, taxonomy, Lycidae, Mexico.

Lycidae (Coleoptera: Elateroidea) es una familia cosmopólita moderadamente diversa con aproximadamente 4000 especies (Bocak y Bocakova, 2008) descritas a nivel global, mientras que para México se reportan 168 (ZaragozaCaballero y Pérez-Hernández, 2014). De acuerdo a la más reciente clasificación, la familia se compone de siete subfamilias: Dexorinae, Calochrominae, Erotinae, Ateliinae, Lycinae, Lyropaeinae y Metriorrhynchinae (Kusy et al. 2019). A la tribu Lycini pertenecen Lycus Fabricius, 1787 y Lycostomus Motchulsky, 1861 (Bocak y Bocakova, 2008). Kleine (1933) reconoce en esa tribu a Celiasis Laporte, 1840, Demosis Waterhouse, 1878, Lipermes Waterhouse, 1879 y Lycus con los subgéneros: Acantholycus Bourgeois, 1883, Hololycus Bourgeois, 1883, Lopholycus Bourgeois, 1883, Lycus s. str., Chlamydolycus Bourgeois, 1883, Haplolycus Bourgeois, 1883, Merolycus Bourgeois, 1883, Neolycus Bourgeois, 1883, Thoracocalon Bourgeois, 1883 y Lycostomus. Para Bocak y colaboradores (comunicación personal), la tribu Lycini cuenta con unas 440 especies en cuatro géneros que son: Celiasis Laporte, 1840 (= Neolycus Bourgeois, 1883), Rhyncheros LeConte, 1881 nombre reinstalado (= Thoracocalon Bourgeios, 1883 syn nov., =Lyconotus Green, 1949 syn. nov.), Lipermes Waterhouse, 1879 y Lycus Fabricius, 1787, incluyendo los subgéneros: Lycostomus, Haplolycyus y Lycus s. str. que se segregan por análisis moleculares y características morfológicas y principalmente, por la configuración de aparato reproductor masculino. Para los mismos autores, en México se encuetran Celiasis y Rhyncheros, géneros no relacionados cercanamente con los géneros: Lycus Afrotropical y el asiático Lycostomus (loc cit). En la revisión que hace Green (1949) de la tribu Lycini de Norteamérica, reconoce los subgéneros Neolycus Burgeois, Lycostomus Motschulsky y Lyconotus Green en el género Lycus Fabricius. Lyconotus fue posteriormente elevado a nivel de género por Zaragoza
(1995). Entre el material que forma parte de la Colección Nacional de Insectos (CNIN), se encontró un ejemplar que no corresponde a alguno de los Lycini hasta ahora descritos y es motivo de esta contribución.

\section{MATERIAL Y MÉTODOS}

Un ejemplar macho procedente de Río Verde, San Luis Potosí se revisó bajo un microoscopio Zeiss Discovery V8. El espécimen fue disectado para extraer el aparato reproductor que fue montado en un triángulo de cartón. Así mismo, con una reglilla micrométrica adaptada al microscopio, se tomaron las medidas de largo y ancho total, largo y ancho del pronoto, largo y ancho de los élitros, distancia interocular, largo y ancho del ojo y los cuatro primeros antenómeros. Las fotografías fueron tomadas utilizando un microoscopio Carl Zeiss estereoscópico Modelo Axio Zoom. V16, equipado con una cámara AxioCam Mrc5. El programa usado que complementa al microscopio fue el ZEN Zeiss eficient navegation. Las medidas se expresan en milímetros. El ejemplar está depositado en la Colección Nacional de Insectos (CNIN) del Instituto de Biología de la Universidad Nacional Autónoma de México.

\section{Lycomesus gen. nov. Zaragoza-Caballero y González- Ramírez \\ http://zoobank.org/7AD5C0F1-D0BF-4442-9EDF- ADB5BEE3F804}

Especie tipo: Lycomesus llorentei sp. nov. aquí designada

Diagnosis. Lycomesus gen. nov. está estrechamente relacionado con Lyconotus. En ambos géneros el prosternón no es carinado y el mesosternón no es prominente, mientras que en Lycus y Lycostomus éstas estructuras están modificadas. Los trocánteres en los machos son espinosos; los ángulos internos de las metacoxas no pronunciados y las espinas metatibiales son iguales. Este último carácter 
los acerca a Lycostomus. Las diferencias mas notables entre ellos, es la configuración del edeago en Lyconotus está formando una escuadra y los lóbulos laterales son ojivales y casi alcanzan la mitad de lo largo del lóbulo medio, en Lycomesus la curvatura ventral alcanza la mitad de la escuadra, en tanto los lóbulos laterales son cuadrados y alcanzan un poco más de la quinta parte de lo largo del lóbulo medio (Fig. 5). En el cuadro 1 se señalan las diferencias y semejanzas entre los géneros de Lycini.

Descripción: Cuerpo alargado, ligeramente ensanchado. Cabeza cubierta por el pronoto; rostro alargado; mandíbulas largas, ligeramente curvadas; antenas de 11 antenómeros; palpos maxilares de cuatro palpómeros, los labiales de tres. Pronoto con el borde anterior redondo, el posterior ondulado, los laterales un tanto convergentes, ángulos posteriores agudos, disco convexo y marcadas explanaciones. Élitros con tres costillas principales, que limitan orificios alveolares; Proesternón lateralmente no comprimido; espiráculos mesotorácicos alargados de forma tubular. Pro- y mesocoxas cortas, separadas; mesosternón plano, no prominente; trocánteres con el ángulo interno agudo; parte interna de las metatibias redondas; espinas metatibiales cortas y anchas. Esternitos abdominales con los ángulos posteriores poco pronunciados, último esternito profundamente excavado, el último con el ápice lanceolado y parcialmente curvado. Edeago con lóbulos laterales cortos, casi cuadrados en vista lateral; lóbulo medio con una curvatura ventral de aproximadamente unos $45^{\circ}$.

Etimología. Lycomesus está formada por lo vocablos griegos. Lykos = lobo; mesus $=$ medio. Este último hace referencia a la curvatura media $\left(45^{\circ}\right)$ del lóbulo medio del edeago, comparándola con la que se presenta en la misma estructura de Lycus y Lycostomus por un lado y Lyconotus por otro.

\section{Lycomesus Ilorentei sp. nov. Zaragoza-Caballero y González-Ramírez Figuras 1-5 \\ http://zoobank.org/B77D2CA2-5B26-455B-A504- 6DE25BC09165}

Descripción. Talla: Largo 8.75. Ancho 2.80. Color negro excepto pronoto y los élitros en sus dos tercios anteriores que son amarillentos (Fig. 1).

Cabeza. Totalmente cubierta dorsalmente por el pronoto, con el rostro largo (Fig. 2), longitud del rostro (0.78) un poco más de una vez y media la distancia interocular (0.50). Ojos semiesféricos, casi tan largos (0.26) como anchos (0.22); distancia interocular (0.50). Frente prominente; distancia interantenal 0.02; Antenas largas, aplanadas, rebasando la mitad del cuerpo, ligeramente serradas; longitud del segundo antenómero (0.13) de menos de la mitad del primero (0.36); longitud individual de los antenómeros 3-10 (0.52), casi tan largos como la distancia interocular; (0.50), el undécimo un poco más largo (0.56). Clípeo ligéramente más largo (0.28) que ancho (0.24), con el borde anterior hendido. Palpos maxilares y labiales con el último palpómero más largo que los dos anteriores reunidos, de ápice redondeado.

Tórax. Pronoto (Fig.1) con una densa pilosidad decumbente; lados curvados, anteriormente convergentes bordes anteriores redondeados, ángulos posteriores agudos, disco muy convexo en su porción anterior, con un surco longitudinal en su porción basal, lados en declive muy pronunciado. Escutelo casi subpentagonal, con el borde posterior truncado. Élitros (Fig.1) ligeramente ensanchados hacia la mitad de la longitud, con cuatro costillas, tres más conspicuas, principalmente la humeral en su parte basal, con pubescencia procumbente de color amarillo. Prosternón con parte posterior ancha, con el borde posterior truncado. Mesosternón casi triangular, con borde posterior truncado, superficie plana. Patas: trocánteres con una proyección muy aguda en su parte interna; tibias y fémures aplanados, acanalados, tibias curvadas, sobre todos las mesotibias; espinas tibiales separadas, cortas, con el ápice acuminado; primer metatarsomero tan largo como los dos siguientes juntos (0.41); uñas simples.

Abdomen. Esternitos con los ángulos posteriores no muy prominentes, el penúltimo con el borde posterior profundamente excavado, último de forma casi triangular y parcialmente curvado. Edeago (Figs. 3-5) con pieza basal un poco más larga que los lóbulos laterales; lóbulos laterales cubriendo la región dorsal, libremente articulados, cortos, (0.31) alcanzando la quinta parte del extenso (1.56) lóbulo medio, que está lateralmente comprimido, un poco ensanchado, y curvado al ápice en vista lateral, la curvatura de aproximadamente $45^{\circ}$ (considerando una línea horizontal hacia la mitad de los lóbulos laterales), orificio medio apical y de forma circular, saco interno no visible. Hembra desconocida.

Etimología. El epíteto llorentei es en reconocimiento a la labor que en el campo de Lepidopterología ha desarrollado el Dr. Jorge Llorente Bousquets.

Holotipo macho etiquetado: México, San Luis Potosí, Río Verde; col noct: 1013 msnm; 21/VII/2008; 2155'35N; 9959'58W; H. Brailovsky y E. Barrera cols. Depositado en la Colección Nacional de Insectos (CNIN) de la Universidad Nacional Autónoma de México. (COL.TIP-03600).

Comentarios finales. Bocaky Bocákova (2008) hacen énfasis en que el conocimiento de los Lycini a nivel global se encuentra en un estado de desorden, el cual se ha tratado de resolver bajo estudios moleculares, sobre todo basados en el muestreo de ejemplares de la región Afroasiática. Esos estudios han sido complementados con el estudio de la variación morfológica, particularmente del aparato reproductor masculino. En tales estudios, las regiones Neartica y Neotropical frecuentemente están representadas por pocas especies que no cubren el universo de la variación morfológica del grupo en caracteres clave como los es el aparato reproductor masculino. Es por esto que consideramos necesario seguir abundando en el reconocimiento de las especies americanas bajo el estudio de esa estructura.

\section{AGRADECIMIENTOS}

Agradecemos a José Luis Navarrete-Heredia por su invitación a esta contribución que rinde un justo reconocimiento al Dr. Jorge Llorente Bousquets, entomólogo comprometidamente dedicado al conocimiento del Orden Lepidoptera; a la Biól. Susana Guzmán por la asistencia técnica en la toma de las fotografías; y a dos revisores por sus comentarios siempre bienvenidos. 


\section{LITERATURA CITADA}

Bocak, L. y M. Bocákova. 2008. Phylogeny and Classification of the Family Lycidae (Insecta: Coleoptera). Annales Zoologici, 58(4): 995-720.

Green, J. 1949. The Lycidae of the United States and Canada: I. The Tribe Lycini (Coleoptera). Transactions of the American Entomological Society, 75(2): 53-70.

Kleine, R. 1933. Coleopterorum Catalugus, pars 128. Lycidae. Junk W. \& S Schenkling (Eds.), W Junk, Berlin, 145 pp.

Recibido: 2 de mayo 2019

Aceptado: 10 de junio 2019
Kusy, D., M. Motyka, Bocek, M. , Masek, M. and Bocák, L. 2019. Phylogenomic analysis resolves the relationships among net-winged beetles (Coleoptera: Lycidae) and reveals the parallel evolution of morphological traits. Systematic Entomology, 1-15. DOI: 10.1111/syen.12363 Zaragoza-Caballero, S. 1995. Cantharoidea (Coleoptera) de México. II. Lycinae de Veracruz. Folia Entomológica Mexicana, 35: 23-84.

Zaragoza-Caballero, S., y C. Pérez-Hernández, 2014. Biodiversidad de cantaroideos (Coleoptera: Elateroidea [Cantharidae, Lampyridae, Lycidae, Phengodidae, Telegeusidae]) en México. Revista Mexicana de Biodiversidad, Suple 85: 279-289.
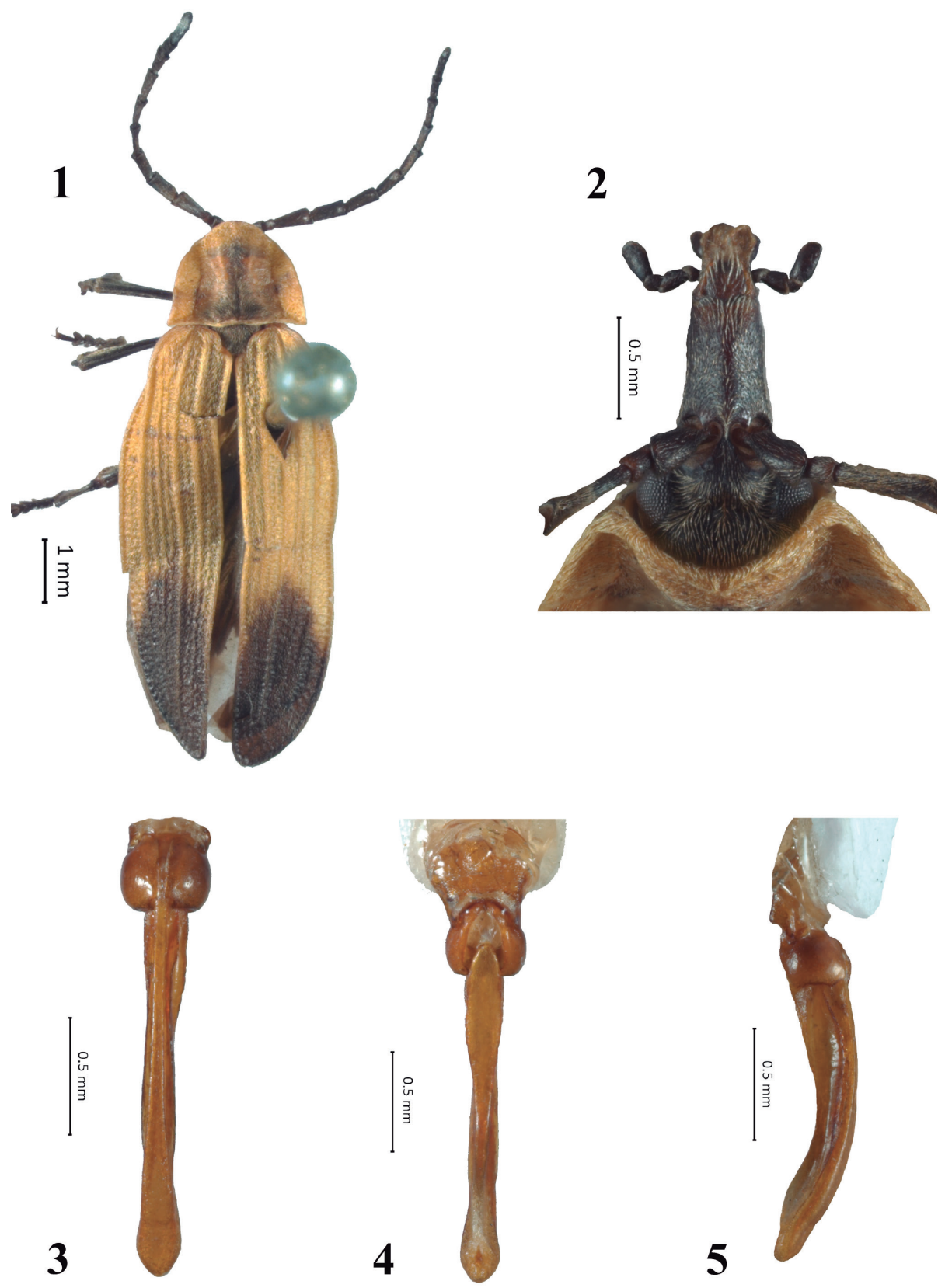

Figuras 1-5 de Lycomesus llorentei gen. et sp. nov. Fig. 1.- Vista dorsal. Fig. 2.- Vista frontal del rostro. Fig. 3-5.- Vista dorsal. lateral y ventral del edeago. 
Cuadro 1. Características distintivas de los géneros Lycus, Lycostomus, Lyconotus y Lycomesus gen. nov. (Coleoptera: Lycidae).

\begin{tabular}{|c|c|c|c|c|c|c|c|}
\hline $\begin{array}{l}\text { Género } \\
\text { Carácter }\end{array}$ & Prosterno & Mesosterno & Trocánteres & Metacoxas & $\begin{array}{c}\text { Espinas } \\
\text { metatibiales }\end{array}$ & $\begin{array}{l}\text { Edeago } \\
\text { Lóbulo } \\
\text { medio }\end{array}$ & $\begin{array}{l}\text { Edeago } \\
\text { lóbulos } \\
\text { laterales }\end{array}$ \\
\hline Lycus & $\begin{array}{l}\text { Lateralmente } \\
\text { comprimido }\end{array}$ & Prominente & Ángulo interno romo & $\begin{array}{l}\text { Ángulo interno } \\
\text { Pronunciado }\end{array}$ & Desiguales & $\begin{array}{c}\text { Curvatura } \\
0^{\circ}\end{array}$ & $\begin{array}{l}\text { Ojivales } \\
\text { cortos }\end{array}$ \\
\hline Lycostomus & $\begin{array}{l}\text { Lateralmente } \\
\text { comprimido }\end{array}$ & Prominente & Ángulo interno romo & $\begin{array}{l}\text { Ángulo interno } \\
\text { Pronunciado }\end{array}$ & Iguales & $\begin{array}{c}\text { Curvatura } \\
0^{\circ}\end{array}$ & $\begin{array}{l}\text { Ojivales } \\
\text { cortos }\end{array}$ \\
\hline Lyconotus & $\begin{array}{c}\text { No } \\
\text { comprimido }\end{array}$ & $\begin{array}{l}\text { No } \\
\text { prominente }\end{array}$ & Ángulo interno agudo & $\begin{array}{l}\text { Ángulo interno } \\
\text { no pronunciados }\end{array}$ & Iguales & $\begin{array}{l}\text { Curvatura } \\
90^{\circ}\end{array}$ & $\begin{array}{l}\text { Ojivales } \\
\text { largos }\end{array}$ \\
\hline $\begin{array}{l}\text { Lycomesus } \\
\text { gen. nov. }\end{array}$ & $\begin{array}{l}\text { No } \\
\text { comprimido }\end{array}$ & $\begin{array}{c}\text { No } \\
\text { prominente }\end{array}$ & Ángulo interno agudo & $\begin{array}{l}\text { Ángulo interno } \\
\text { no pronunciado }\end{array}$ & Iguales & $\begin{array}{c}\text { Curvatura } \\
45^{\circ}\end{array}$ & $\begin{array}{c}\text { Cuadrados } \\
\text { cortos }\end{array}$ \\
\hline
\end{tabular}

